\title{
La comunicación científica en Uruguay: estudio de las publicaciones de los investigadores activos del Sistema Nacional de Investigadores (2009-2010)
}

\author{
Scholarly communication in Uruguay: Study of \\ publications of active researchers from the \\ National System of Researchers (2009-2010)
}

\author{
Paola PICCO' (in memoriam) \\ Natalia AGUIRRE-LIGÜERA' \\ Juan MALDINI² \\ Lucía SIMÓN' \\ Patricia PETROCCELLI ${ }^{1}$ \\ Exequiel FONTANS ${ }^{1}$ \\ José FAGER ${ }^{1}$ \\ María Gladys CERETTA`
}

\section{Resumen}

Se estudia el fenómeno de la comunicación científica en Uruguay considerando las publicaciones declaradas en los Currícula Vitae por parte de los investigadores que integran el Sistema Nacional de Investigadores de la Agencia Nacional de Investigación e Innovación de Uruguay. Se construye una base de datos ad-hoc, en la que se registran los datos relativos a las siguientes variables: área de conocimiento, tipo de publicación, tipo de responsabilidad, idioma, lugar de publicación, soporte, arbitraje e indización. El análisis de los datos se realiza a partir de una aproximación cuantitativa, con métodos estadísticos descriptivos. Los datos se procesan mediante un software para análisis estadístico. En consonancia con la literatura se encuentra que los patrones de comunicación varían de acuerdo al área de conocimiento. Se concluye que los Currícula Vitae, a pesar de sus limitaciones, son una fuente rica para abordar el estudio de la producción científica en su conjunto, y que estos trabajos son complementarios a los estudios bibliométricos realizados a partir de bases de datos internacionales que permiten estudiar otros aspectos.

Palabras Ilave: Comunicación científica. Currículum vitae. Sistema Nacional de Investigadores. Uruguay.

\footnotetext{
Abstract

Scholarly communication in Uruguay was studied considering the publications reported in the researchers' curricula vitae from the National System of Researchers of the National Agency for Research and Innovation of Uruguay. An ad-hoc database was developed, where data relative to variables such as field of knowledge, kind of publication, type of responsibility, place of publication, support,

1 Universidad de la República, Facultad de Información y Comunicación, Instituto de Información. Calle: Emilio Frugoni, 1427, 11200, Montevideo, Uruguay. Correspondencia a nombre de/Correspondence to: N. AGUIRRE-LIGÜERA.E-mail:<naguirre@eubca.edu.uy>.

2 Universidad de la República, Facultad de Información y Comunicación, Programa de Desarrollo Académico de la Información y la Comunicación. Montevideo, Uruguay.

Recibido el dia 27/5/2013, re-apresentado em 12/10/2013 y aceptado para su publicación el 11/12/2013.
} 
language, peer review and indexing were registered. A quantitative data analysis was carried out with descriptive statistical methods, using a software for statistical analysis. In accordance to the literature, it has been found that communication patterns vary depending on the field of knowledge. It is concluded that the curricula vitae, regardless of their limitations, are a rich source to approach the study of scientific production globally and complement bibliometric studies through international databases which allow the study of other aspects.

Keywords: Scholars communication. Curriculum vitae. National System of Researchers. Uruguay.

\section{Introducción}

La comunicación científica, actividad inherente a la ciencia, se basa en la necesidad de difundir los resultados de las investigaciones al resto de la comunidad para que sean conocidos, validados y utilizados en la generación de nuevo conocimiento. En su clásica definición Borgman (1989) sostiene que puede entenderse como la forma en que se comunican y difunden los resultados de investigaciones en cualquier disciplina, ya sea a través de canales formales o informales.

La producción científica se configura como espejo de la ciencia y de la comunidad científica de un país y de una disciplina (Targino, 2010). El investigador generalmente no persigue beneficios económicos inmediatos con sus publicaciones y no recibe remuneración por parte de los editores por su trabajo sino que, como plantea Maltrás Barba (2003), la publicación se constituye en canal reivindicativo de la prioridad del investigador respecto a los hallazgos, aporta a su reconocimiento por parte de la comunidad y al incremento de su prestigio.

El desarrollo de la ciencia en cualquier país y especialidad se ve condicionado por la posibilidad de acceder al conocimiento previamente registrado, dada la tradición acumulativa del conocimiento científico ya señalada por Bernal (1967). En este sentido, Russell (2001) sostiene que es tan importante comunicar los resultados como acceder a los hallazgos de investigaciones previas, para formular propuestas y metodologías de investigación.

Una de las principales manifestaciones de esta actividad es la publicación de artículos o papers en revistas científicas (Sancho, 1990). La aparición de las mismas se remonta hacia finales del siglo XVII y sustituye la correspondencia entre los miembros de las sociedades científicas (López-Borrul, 2012). El artículo en revistas científicas arbitradas es el tipo de documento privilegiado en el modelo tradicional de comunicación científica, lo que se refleja en los estudios que tienen como fuente las bases de datos internacionales que principalmente indizan estos documentos. Maltrás Barba (2003, p.20) denomina a los artículos en revistas arbitradas como publicaciones oficiales, planteando que operan como "Filtro protector que salvaguarda la autoridad y prestigio de la ciencia [generando un] corpus de conocimiento público y consensuado". Desde esta perspectiva todo lo que se publica por otros canales quedaría fuera de ese corpus.

Estudios anteriores revelan que los mecanismos que utilizan los investigadores para comunicar sus resultados varían de acuerdo a las distintas áreas de conocimiento, e incluso a las disciplinas que las conforman (Van Raan, 1997; Glanzel \& Schoepflin, 1999; Bordons et al., 2010; Gantman, 2011). Estas diferencias responden a las características del objeto de estudio, las metodologías y las dinámicas referidas al consumo de información científica instaladas en cada disciplina, que afectan la forma en que los investigadores comunican sus aportes (Russell \& Liberman, 2002).

En la literatura se encuentran conceptualizaciones que enfatizan en distintos elementos para definir qué se entiende por modelo de comunicación científica. Están quienes refieren al ciclo de comunicación o el recorrido que transita el conocimiento para llegar a ser publicado y citado. En esta línea Garvey y Griffith (1972) desarrollan su tradicional modelo distinguiendo entre medios formales e informales de comunicación. Según éste los resultados preliminares de una investigación son comunicados a través de medios informales (comunicaciones interpersonales, seminarios, coloquios, conferencias). A medida que la investigación avanza y obtiene el respaldo de la comunidad se preparan artículos para ser publicados en revistas científicas arbitradas. Con el paso del tiempo se espera que estos artículos sean incluidos en revisiones y fuentes secundarias. 
Este modelo ha sido retomado y actualizado por distintos autores. Hurd (2000) analiza el impacto de las Tecnologías de la Información y la Comunicación (TIC) y propone un modelo basado en medios digitales. Introduce la idea de que este entorno propicia un cambio en los roles de los actores tradicionales, por ejemplo las bibliotecas, quienes comienzan a desarrollar nuevos productos y servicios. Esta autora incorpora la noción de acceso al conocimiento científico, introduciendo al modelo distintas herramientas de control bibliográfico que permiten la organización y recuperación del conocimiento, como son los catálogos, los servicios de citación, entre otros.

Asimismo Björk (2005) presenta un modelo jerárquico que focaliza en particular en los artículos publicados en revistas arbitradas, agregando como actores involucrados no solo a los autores, sino también a revisores, editores, impresores, lectores, bibliotecarios, así como añade las tareas que realizan: redacción, revisión, impresión, edición, distribución, archivo, recuperación, lectura, entre otras.

Están también quienes conceptualizan al modelo de comunicación científica en relación a la distribución y posibilidad de acceso a la literatura científica. Aquí aparece el modelo de acceso abierto en aparente oposición al modelo comercial tradicional monopolizado por las grandes editoriales.

\begin{abstract}
El sistema de archivos abiertos no solo determina profundas transformaciones a nivel tecnológico sino que se presenta como un movimiento sociocientífico tendiente a cambiar completamente el modelo vigente de la comunicación académica y el sistema científico de forma más amplia. Se estaría frente a un cambio de paradigma de los procesos de comunicación especializada, priorizando la divulgación antes que el 'negocio' de la información (Gómez \& Arias, 2002, p.100).
\end{abstract}

En este trabajo se presentan resultados de la primera etapa del proyecto de Investigación y Desarrollo $(I+D)$ Modelo de repositorio institucional como alternativa para el desarrollo social, científico y tecnológico del País, de la Universidad de la República (Uruguay). El mismo se propone identificar los patrones de comunicación que siguen los investigadores uruguayos para difundir los resultados de sus actividades y analizar en qué medida ese conocimiento registrado está visibley disponible libremente para sus instituciones de afiliación. Partiendo de este estado de situación se apunta a evaluar la viabilidad de construir un modelo de repositorio que propenda al acceso abierto al conocimiento científico originado en el País, particularmente aquel generado con fondos públicos. Este modelo de repositorio deberá considerar, entre otros aspectos, los patrones de comunicación identificados.

En el marco de este proyecto se considera que el acceso abierto no constituye un nuevo modelo de comunicación científica, en la medida que no atenta contra las bases del modelo tradicional, sino que se trata de una filosofía que convive con éste y lo que busca es democratizar el acceso al conocimiento registrado, en oposición al modelo de negocios de los grandes grupos editoriales. En relación a las dos vías propuestas para su concreción, la vía dorada (publicación en revistas de acceso abierto) se mantiene fiel al sistema de publicación en revistas científicas arbitradas, pero cambia los mecanismos de acceso a las mismas. Mientras que la vía verde (autoarchivo en repositorios digitales) no tiene como objetivo la publicación sino la difusión de los trabajos en repositorios de acceso abierto; esos trabajos pueden haber sido publicados tanto en revistas de suscripción como de vía dorada (Miguel et al., 2012).

Este estudio se centra en el análisis de las publicaciones de los investigadores nacionales, dejando de lado otros procesos involucrados, como por ejemplo el acceso, que será objeto de otra publicación por parte este equipo.

\section{Situación nacional}

Uruguay viene impulsando, desde 2005, una batería de políticas nacionales destinadas a la promoción de la Ciencia y la Tecnología (CyT). La Agencia Nacional de Investigación e Innovación (ANII) ejecuta y articula las políticas públicas de CyT y tuvo entre sus cometidos la creación del Sistema Nacional de Investigadores (SNI) con el objetivo de identificar, consolidar y expandir la comunidad científica nacional. También puso en funcionamiento el portal Timbó <http://www.timbo. org.uy/>, que brinda acceso a la comunidad educativa y científica a bases de datos científicas, referenciales y a texto completo, por las que el país paga dos millones de dólares anuales (Uruguay, 2012). 
A partir de entrevistas realizadas a distintos actores del ámbito político-educativo se puede constatar que está presente en la agenda política la preocupación por la problemática del acceso a la información científica. Sin embargo no se han detectado antecedentes de investigación que tomen como objeto el fenómeno de la comunicación científica a nivel nacional, con excepción de los trabajos de Fernández et al. (2005) y de Aguirre-Ligüera (2009-2011) en los que se consideran las publicaciones del País recogidas en la Web of Science (WoS).

El éxito de la instrumentación de políticas tendientes a generar mecanismos de acceso abierto estará condicionado por el conocimiento de este fenómeno. Esta problemática no se limita a las dificultades para acceder a los contenidos sino también a la ausencia de mecanismos de control bibliográfico que den cuenta de la producción científica nacional, con excepción de algunas iniciativas aisladas y limitadas a disciplinas específicas.

\section{Métodos}

Se analizan los patrones de publicación seguidos por los investigadores activos, registrados y categorizados en el SNI, agrupados en las seis áreas definidas por la ANII. Se toma como objeto de estudio la producción científica correspondiente a los años 2009 y 2010 declarada en sus Currícula Vitae (CV). La unidad de análisis está constituida por las publicaciones.

La fuente de datos para el estudio la constituyen los CV ingresados en el sistema automatizado de currículum vitae de Uruguay, denominado CVuy. El mismo está basado en el CV-LAC (Currículum Vitae Latinoamericano) heredero del CV Lattes desarrollado en Brasil. El CVuy es el instrumento de evaluación que permite determinar si el investigador ingresa al sistema, en qué categoría y en cuál de las seis áreas se encuentra categorizado. De este modo el CVuy constituye una fuente de información de gran utilidad en un país que no cuenta con bases de datos que registren la producción científica nacional. Asimismo estudios anteriores insisten en que los sesgos, sobre todo temático e idiomático, de las bases de datos internacionales comerciales hacen que no resulten las mejores fuentes cuando se trata de estudiar la producción científica de ciertos países y áreas del conocimiento (Fernández et al., 2005; Sanz Casado \& Conforti, 2005; De Filippo et al., 2011).

La fuente de datos (CVuy) presenta ciertas limitaciones señaladas en la bibliografía (Dietzet al., 2000), porque contiene información auto-reportada, es decir, susceptible a errores intencionados o no, y a información faltante, inconsistente o no actualizada. Sin embargo, de la revisión bibliográfica también surge que se trata de una fuente rica y muchas veces única, aunque de uso bastante reciente en estudios por parte de gestores e investigadores de la ciencia (Cañibano \& Bozeman, 2009). Para esta investigación es la única fuente disponible que recoge toda la producción de los científicos categorizados en el SNI.

El análisis de los datos se realiza a partir de una aproximación cuantitativa, con métodos estadísticos descriptivos. Los datos se procesan mediante el software estadístico Statistical Package for the Social Sciences (SPSS) v.19 en español, de la empresa IBM.

La recolección de datos se lleva a cabo entre mayo y setiembre de 2011, desde el sitio web del SNI < http:// www.anii.org.uy/web/paginas/cvuy> consultando todos los CV y cargando una base de datos propia que incluye las siguientes variables y categorías, previamente codificadas y mutuamente excluyentes.

- Área de conocimiento, esta variable tiene 6 categorías definidas por el SNI: Humanidades, Ciencias Agrícolas, Ciencias Naturales y Exactas, Ciencias Médicas y de la Salud, Ingeniería y Tecnología y Ciencias Sociales.

- Tipo de publicación, sus categorías son: artículo, monografía, capítulo de monografía, ponencia (publicadas en actas de eventos científicos), producción técnica (incluye patentes, informes técnicos, y otros), artículos de difusión (publicados en prensa y otras publicaciones no especializadas). Las categorías son tomadas del CVuy.

- Tipo de responsabilidad, las categorías que asume son: individual, colectiva (más de un autor) y editor/ compilador.

- Lugar de publicación, las categorías previstas son: Uruguay, Mercosur (sin Uruguay), Resto de América Latina, Estados Unidos y Canadá, Europa, otros. 
- Soporte, sus categorías son: papel, digital, otros y sin dato.

- Idioma, puede asumir las categorías: español, inglés y otros.

- Arbitraje, se aplica solo a artículos, sus categorías son: arbitrado y no arbitrado.

- Indización, se aplica solo a artículos, sus categorías son: indizado y no indizado. Entendiendo por indizados aquellos artículos publicados en revistas recogidas por Web of Science, Scopus o por ambas, ya que son las que recoge en CVuy.

El análisis de los datos se hace a partir de la variable área de conocimiento, tomando en cuenta las diferencias de comportamiento entreáreas mencionadas en la introducción. En cada área se eliminan los registros duplicados reportados al sistema CVuy por más de un investigador, producto de la colaboración entre investigadores de la misma área.

Considerar toda la producción científica en sus distintos tipos documentales, tiene la ventaja de permitir identificar los patrones imperantes en el proceso de difusión y publicación de los resultados, sin privilegiar un tipo de documento sobre otro. Esto habilita el análisis de algunas publicaciones que seguramente no son recogidas por ninguna fuente de datos nacional o internacional. Sin embargo, se presume fundamental analizar particularmente aquella parcela de la producción científica que cumple con ciertos estándares de calidad, aquella que para ser publicada se sometió previamente a un proceso de revisión por pares. Por lo anterior, se divide el análisis en una primera parte de carácter general y luego se consideran específicamente los artículos de revistas científicas.

\section{Resultados y Discusión}

Los resultados están en consonancia con la idea presente en la bibliografía de que las publicaciones en las distintas áreas de conocimiento no presentan un comportamiento homogéneo.

Se identifican 1.252 investigadores que declaran haber publicado 8.808 publicaciones, en el período 20092010. Se encuentran 1.748 publicaciones ingresadas más de una vez al sistema, por distintos investigadores, producto de la colaboración entre al menos dos investigadores de la misma área de conocimiento. $\mathrm{Al}$ eliminar estos duplicados se obtiene un total de 7.060 publicaciones. Estas distribuciones se aprecian en la Tabla 1.

En la representación de cada área a partir del número de investigadores se destaca Ciencias Naturales y Exactas con más de un tercio del total de investigadores del sistema. Por esto no sorprende que sea el área que más produce en términos absolutos, aportando más de un cuarto de las publicaciones. La fuerte presencia de esta área en el SNI se explicaría, al menos en parte, por la aplicación sostenida de algunas políticas fuertes para el desarrollo de la misma durante más de 25 años, como el Programa de Desarrollo de las Ciencias Básicas (PEDECIBA).

La búsqueda de similitudes y divergencias entre las publicaciones de las distintas áreas a partir de la Tabla 2 arroja comportamientos similares entre Ciencias Sociales y Humanidades en las cinco variables consideradas, formando una dupla suficientemente homogénea. Las otras cuatro áreas se diferencian sustancialmente de éstas y se parecen bastante entre sí.

Tabla 1. Investigadores y publicaciones por área de conocimiento.

\begin{tabular}{|c|c|c|c|c|}
\hline \multirow{2}{*}{ Áreas } & \multicolumn{2}{|c|}{ Investigadores } & \multicolumn{2}{|c|}{ Publicaciones } \\
\hline & Frecuencia absoluta & Porcentaje & Frecuencia absoluta & Porcentaje \\
\hline Ciencias Agrícolas & 185 & 14,8 & 1.572 & 22,2 \\
\hline Ciencias Médicas y de la Salud & 167 & 13,3 & 1.038 & 14,7 \\
\hline Ingeniería y Tecnología & 126 & 10,1 & 556 & 7,9 \\
\hline Ciencias Naturales y Exactas & 422 & 33,7 & 1.826 & 25,9 \\
\hline Ciencias Sociales & 244 & 19,5 & 1.407 & 19,9 \\
\hline Humanidades & 108 & 8,6 & 661 & 9,4 \\
\hline Total & 1.252 & 100,0 & 7.060 & 100,0 \\
\hline
\end{tabular}

Fuente: Sistema CVuy. Elaboración propia (2013). 
Tabla 2. Publicaciones (en porcentaje) por área de conocimiento, tipo de publicación, tipo de responsabilidad, lugar de publicación, soporte e idioma.

\begin{tabular}{|c|c|c|c|c|c|c|c|}
\hline $\begin{array}{l}\text { Áreas de } \\
\text { conocimiento }\end{array}$ & & $\begin{array}{l}\text { Ciencias } \\
\text { Agrícolas }\end{array}$ & $\begin{array}{l}\text { Ciencias Médicas } \\
\text { y de la Salud }\end{array}$ & $\begin{array}{l}\text { Ingeniería y } \\
\text { Tecnología }\end{array}$ & $\begin{array}{c}\text { Ciencias Naturales } \\
\text { y Exactas }\end{array}$ & $\begin{array}{l}\text { Ciencias } \\
\text { Sociales }\end{array}$ & Humanidades \\
\hline Variables & Categorías & & & 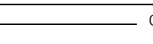 & 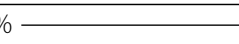 & 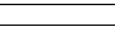 & \\
\hline \multirow[t]{7}{*}{ Tipo de publicación } & Artículo & 28,1 & 36,7 & 30,8 & 44,9 & 25,9 & 20,4 \\
\hline & Monografía & 2,4 & 0,7 & 1,4 & 2,1 & 14,3 & 16,7 \\
\hline & $\begin{array}{l}\text { Capítulo } \\
\text { Monografía }\end{array}$ & 6,6 & 6,1 & 4,3 & 8,2 & 30,5 & 29,2 \\
\hline & Ponencia & 52,5 & 54,1 & 62,1 & 38 & 28,4 & 19,8 \\
\hline & Producción técnica & 4,6 & 1,1 & 0,5 & 2,7 & 0,4 & 0,9 \\
\hline & $\begin{array}{l}\text { Artículo de } \\
\text { difusión }\end{array}$ & 5,8 & 1,3 & 0,9 & 4,1 & 0,5 & 13,0 \\
\hline & Total & 100,0 & 100,0 & 100,0 & 100,0 & 100,0 & 100,0 \\
\hline \multirow{4}{*}{$\begin{array}{l}\text { Tipo de } \\
\text { responsabilidad }\end{array}$} & Individual & 8,5 & 7,2 & 9,9 & 9,2 & 56,4 & 70,2 \\
\hline & Colectiva & 90,5 & 92,7 & 90,1 & 89,8 & 40,4 & 22,5 \\
\hline & Editor/Compilador & 1,0 & 0,1 & 0,0 & 1,0 & 3,2 & 7,3 \\
\hline & Total & 100,0 & 100,0 & 100,0 & 100,0 & 100,0 & 100,0 \\
\hline \multirow[t]{7}{*}{ Lugar de publicación } & Uruguay & 48,5 & 34,8 & 18,0 & 25,6 & 48,0 & 56,1 \\
\hline & $\begin{array}{l}\text { Mercosur sin } \\
\text { Uruguay }\end{array}$ & 14,9 & 12,0 & 28,2 & 14,1 & 19,5 & 16,9 \\
\hline & $\begin{array}{l}\text { Resto de América } \\
\text { Latina }\end{array}$ & 7,0 & 9,6 & 4,5 & 6,1 & 11,5 & 9,7 \\
\hline & $\begin{array}{l}\text { Estados Unidos y } \\
\text { Canadá }\end{array}$ & 8,6 & 14,7 & 14,9 & 18,7 & 4,7 & 3,6 \\
\hline & Europa & 17,2 & 25,1 & 31,5 & 31,1 & 13,9 & 12,7 \\
\hline & Otros/Sin dato & 3,8 & 3,8 & 2,9 & 4,4 & 2,4 & 1,0 \\
\hline & Total & 100,0 & 100,0 & 100,0 & 100,0 & 100,0 & 100,0 \\
\hline \multirow[t]{4}{*}{ Idioma } & Español & 62,6 & 47,9 & 36,0 & 34,4 & 84,7 & 91,7 \\
\hline & Inglés & 35,1 & 51,8 & 62,9 & 64,8 & 13,2 & 5,4 \\
\hline & Otros & 2,3 & 0,3 & 1,1 & 0,8 & 2,1 & 2,9 \\
\hline & Total & 100,0 & 100,0 & 100,0 & 100,0 & 100,0 & 100,0 \\
\hline \multirow[t]{5}{*}{ Soporte } & Papel & 64,0 & 65,2 & 49,4 & 67,5 & 77,4 & 75,7 \\
\hline & Electrónico & 30,3 & 32,3 & 44,1 & 26,8 & 22,2 & 23,4 \\
\hline & Otros & 0,6 & 2,5 & 0,2 & 1,0 & 0,3 & 0,3 \\
\hline & Sin dato & 5,1 & 0,0 & 6,3 & 4,7 & 0,1 & 0,6 \\
\hline & Total & 100,0 & 100,0 & 100,0 & 100,0 & 100,0 & 100,0 \\
\hline
\end{tabular}

Fuente: Sistema CVuy. Elaboración propia (2013).

Esto va en la misma línea de la literatura especializada a nivel internacional (Glanzel \& Schoepflin, 1999; Hicks, 1999), que distingue claramente el tratamiento de la producción de las Ciencias Sociales y Humanas de otras áreas científicas.

\section{Ciencias Sociales y Humanidades}

La dupla Sociales y Humanidades se comporta parecido en cuanto al tipo de publicación. El capítulo de monografía es la categoría más representada rondando 
el 30\% de las publicaciones, seguida por los artículos con poco más del 20\%, y un peso mayor en las Ciencias Sociales que en las Humanidades. Sin embargo, al sumar las categorías capítulo de monografía y monografía, en función de sus semejanzas, representan cerca del 45\% despegándose ampliamente de las otras categorías. Hicks (1999) sostiene que la literatura de las Ciencias Sociales tiene un carácter más fragmentado, debido a que se dan menos consensos entre los investigadores y conviven distintos paradigmas. Esto suele vincularse con la mayor publicación de monografías, en contraposición a la señal de consenso que significaría la publicación de artículos. En la misma línea, Glanzel y Schoepflin (1999) sostienen que en estas áreas los modelos estándar de comunicación científica, basados en publicaciones seriadas, requieren revisiones sustantivas para ser aplicados.

Para la variable tipo de responsabilidad, más de la mitad de las publicaciones son producto del trabajo individual de un investigador. Rovira (2006) afirma que este comportamiento autoral es otra de las características que distingue a estas áreas. Sin embargo, cabe diferenciar que mientras en las Ciencias Sociales la autoría individual alcanza poco más de la mitad y la colectiva aproximadamente un 40\%, la relación en Humanidades es de un $70 \%$ contra un $22 \%$. Asimismo la categoría editor/compilador solo consigue cierto peso en estas áreas, muy posiblemente por el alto porcentaje de monografías declarado.

Tabla 3. Artículos (en porcentaje) por área de conocimiento, arbitraje, indización, tipo de responsabilidad, lugar de publicación e idioma.

\begin{tabular}{|c|c|c|c|c|c|c|c|}
\hline $\begin{array}{l}\text { Áreas de } \\
\text { conocimiento }\end{array}$ & & $\begin{array}{l}\text { Ciencias } \\
\text { Agrícolas }\end{array}$ & $\begin{array}{c}\text { Ciencias Médicas } \\
\text { y de la Salud }\end{array}$ & $\begin{array}{l}\text { Ingeniería y } \\
\text { Tecnología }\end{array}$ & $\begin{array}{c}\text { Ciencias Naturales } \\
\text { y Exactas }\end{array}$ & $\begin{array}{l}\text { Ciencias } \\
\text { Sociales }\end{array}$ & Humanidades \\
\hline Variables & Categorías & & & & & & \\
\hline \multirow[t]{3}{*}{ Arbitraje } & Arbitrada & 90,7 & 98,4 & 95,9 & 98,0 & 67,9 & 69,6 \\
\hline & No arbitrada & 9,3 & 1,6 & 4,1 & 2,0 & 32,1 & 30,4 \\
\hline & Total & 100,0 & 100,0 & 100,0 & 100,0 & 100,0 & 100,0 \\
\hline \multirow[t]{3}{*}{ Indización } & Indizada & 66,1 & 82,2 & 77,8 & 91,0 & 16,8 & 14,1 \\
\hline & No indizada & 33,9 & 17,8 & 22,2 & 9,0 & 83,2 & 85,9 \\
\hline & Total & 100,0 & 100,0 & 100,0 & 100,0 & 100,0 & 100,0 \\
\hline \multirow{3}{*}{$\begin{array}{l}\text { Tipo de } \\
\text { responsabilidad }\end{array}$} & Individual & 4,8 & 5,8 & 8,2 & 5,9 & 56,9 & 85,2 \\
\hline & Colectiva & 95,2 & 94,2 & 91,8 & 94,1 & 43,1 & 14,8 \\
\hline & Total & 100,0 & 100,0 & 100,0 & 100,0 & 100,0 & 100,0 \\
\hline \multirow[t]{7}{*}{ Lugar de publicación } & Uruguay & 18,1 & 7,9 & 12,3 & 3,7 & 37,1 & 37,0 \\
\hline & $\begin{array}{l}\text { Mercosur sin } \\
\text { Uruguay }\end{array}$ & 14,5 & 5,0 & 6,4 & 6,6 & 20,6 & 16,3 \\
\hline & $\begin{array}{l}\text { Resto de América } \\
\text { Latina }\end{array}$ & 4,5 & 7,3 & 4,1 & 2,9 & 13,7 & 12,6 \\
\hline & $\begin{array}{l}\text { Estados Unidos y } \\
\text { Canadá }\end{array}$ & 15,2 & 26,5 & 24,0 & 28,5 & 5,8 & 11,9 \\
\hline & Europa & 40,3 & 48,3 & 52,6 & 54,5 & 21,2 & 21,5 \\
\hline & Otros/Sin dato & 7,4 & 5,0 & 0,6 & 3,8 & 1,6 & 0,7 \\
\hline & Total & 100,0 & 100,0 & 100,0 & 100,0 & 100,0 & 100,0 \\
\hline \multirow[t]{4}{*}{ Idioma } & Español & 28,5 & 19,2 & 20,5 & 7,1 & 76,1 & 85,2 \\
\hline & Inglés & 67,9 & 80,8 & 79,5 & 92,4 & 21,4 & 11,1 \\
\hline & Otros & 3,6 & 0,0 & 0,0 & 0,5 & 2,5 & 3,7 \\
\hline & Total & 100,0 & 100,0 & 100,0 & 100,0 & 100,0 & 100,0 \\
\hline
\end{tabular}

Fuente: Sistema CVuy. Elaboración propia (2013). 
El lugar de publicación más representado es Uruguay, alcanzando prácticamente la mitad de la producción científica de la dupla. Este aspecto se relaciona con la publicación de monografías y capítulos y con la tendencia de estas áreas a investigar sobre temas locales de escaso interés internacional (Hicks, 1999). Al sumar las categorías que corresponden a trabajos publicados en América Latina, en Ciencias Sociales alcanzan el 79\%, mientras que en Humanidades este porcentaje sube al $82 \%$. Se confirma que aunque"No son muchos los estudios empíricos que posibilitan el análisis comparativo, algunos trabajos sugerirían que la tendencia a publicar en el ámbito latinoamericano es común entre científicos [sociales] de la región"(Gantman, 2011, p.423).

Más de tres cuartos de las publicaciones se registran en soporte papel y una proporción aún mayor se publica en idioma español. Con relación a la variable idioma es oportuno indicar algunos matices, mientras que en Humanidades solo el $5 \%$ se publica en inglés, en Ciencias Sociales el porcentaje asciende a un 13\%, esto podría deberse a que dentro de esta área conviven disciplinas que se comportan de manera diferente al resto, por ejemplo Economía o Psicología (Gantman, 2011). Asimismo ambas áreas, conjuntamente con Ciencias Agrícolas, son las que cuentan con más publicaciones en otros idiomas, entre los que destacan portugués, italiano y francés.

En la Tabla 3 se considera separadamente la porción de publicaciones conformada por artículos en revistas científicas. Corresponde en primer lugar analizar el comportamiento de dos variables, arbitraje e indización, que solo aplican a este tipo documental. En ambas áreas más de dos tercios del total de artículos se publican en revistas arbitradas, pero los artículos indizados apenas rondan el 15\%. Esto da cuenta de la poca visibilidad internacional de muchas revistas arbitradas en las que los investigadores de estas áreas publican. Esto podría explicarse por la baja visibilidad de las revistas científicas editadas en América Latina (Fernández et al., 2005).

En segundo lugar es interesante observar el comportamiento de algunas variables para los artículos y su relación con el total de la producción. La variable tipo de responsabilidad en los artículos no presenta diferencias significativas con el conjunto de todas las publicaciones. Sin embargo, en lugar e idioma de publicación se encuentra un incremento de la presencia del inglés en detrimento del español, al mismo tiempo que disminuye el peso de América Latina como región de publicación y crecen considerablemente otras como Estados Unidos y Canadá y, principalmente, Europa.

\section{Ciencias Agrícolas, Médicas y de la Salud, Naturales y Exactas e Ingeniería y Tecnología}

El cuarteto formado por las Ciencias Agrícolas, Ciencias Médicas y de la Salud, Ingeniería y Tecnología, Ciencias Naturales y Exactas no es tan parejo como las Ciencias Sociales y Humanidades, aunque sus regularidades y su relativo apego al modelo tradicional permiten considerarlas en conjunto.

Para la variable tipo de publicación la categoría ponencias publicadas corresponde a algo más de la mitad de las publicaciones en las áreas Agrícola y Médica, mientras que en Ingeniería y Tecnología alcanza el 62\%. En los tres casos la categoría que le sigue es el artículo, rondando el 30\%. Siguiendo el modelo de Garvey y Griffith (1972) se podría asumir, aunque la metodología utilizada no permite afirmarlo, que la prominencia de ponencias responde a que este tipo de documentos es utilizado para circular y discutir los hallazgos entre pares, buscando su validación para luego ser publicados en artículos de revistas arbitradas. Algunas de estas ponencias no alcanzarían el apoyo o interés para generar un artículo. Por su parte en las Ciencias Naturales y Exactas es el artículo quien tiene más peso alcanzando casi el $45 \%$, seguido por las ponencias con 38\%.

La importancia del artículo para estas cuatro áreas era de esperar. Esta tendencia está en consonancia con la detectada por Russell y Liberman (2002) para los investigadores de una universidad mexicana, salvando las distancias metodológicas. Estas investigadoras afirman que los artículos predominan en las áreas Exactas, Naturales y Aplicadas distanciándose de las Humanidades y Sociales, aunque en su investigación no consideran las ponencias.

Las variables idioma y el lugar de publicación se presentan estrechamente relacionadas. Para ambas se 
encuentran ciertas similitudes entre las áreas Médica y Agrícola, que publican en idioma español cerca de la mitad y del 60\% de sus trabajos respectivamente. Las Ciencias Médicas publican un tercio en Uruguay, y sumando con el resto de Latinoamérica, esta proporción sube a poco más de la mitad de los trabajos. Las Ciencias Agrícolas por su parte publican en Uruguay prácticamente la mitad y en la región latinoamericana aproximadamente el 70\% del total de sus trabajos. La distribución de las dos variables condice con la relación antes mencionada. Más del $90 \%$ de los trabajos en español son publicados en Latinoamérica en ambas áreas.

Sin embargo, en las Ciencias Médicas poco más del 50\% de la producción es en inglés. Esta cifra la acerca a las Ciencias Naturales e Ingeniería, aunque en las últimas supera el 60\% mientras que sólo un 30\% se produce en español. La hegemonía del inglés está evidentemente vinculada con la publicación en países angloparlantes y en revistas científicas internacionales.

El soporte papel supera ampliamente las otras categorías, excepto en el área Ingeniería y Tecnología, en la cual este soporte y el digital son similares, sin alcanzar diferencias tan importantes como en Humanidades y Sociales. En este aspecto, se encontraron indicios de que ante publicaciones en formato papel y electrónico, los autores reportan el primero.

La variable tipo de responsabilidad presenta valores similares dentro del cuarteto. La enorme mayoría (en torno al 90\%) de los trabajos son de responsabilidad colectiva, es una diferencia muy significativa respecto a la autoría individual que prima en Humanidades y Sociales, confirmando las tendencias detectadas en la literatura.

En relación a los artículos más del 90\% son arbitrados en las cuatro áreas consideradas, alcanzando casi la totalidad en Ciencias Médicas y de la Salud y en Naturales y Exactas, como evidencia la Tabla 3. La indización es también muy significativa, aunque se distribuye heterogéneamente entre las áreas yendo desde el 66\% en Agrícolas hasta el 91\% en Naturales y Exactas, lo que habla de la mayor visibilidad de la producción en estas áreas. Este tipo de documento mantiene el patrón general para todas las publicaciones con más del 90\% de autoría colectiva. En relación al idioma predomina ampliamente el inglés en todas las áreas. Para el lugar de publicación las categorías reunidas en la región latinoamericana oscilan en un rango que va desde el 13\% en Naturales y Exactas a un 37\% en Médicas. Destacan los artículos publicados en Europa representando aproximadamente la mitad del total, con excepción del área Agrícola, y los publicados en la región norteamericana con un cuarto.

Por último, la comunicación pública de los resultados de investigación científica (muchas veces denominada divulgación científica) no ha sido objeto frecuente de estudio desde la Ciencia de la Información en Uruguay. Aunque el fenómeno trasciende los objetivos de este trabajo, es interesante señalar la baja frecuencia de aparición de la categoría artículos de difusión. La misma se distribuye gradualmente en un rango que va desde el $0.5 \%$ en Ciencias Sociales hasta el 5.8\% en Ciencias Agrícolas, con un pico de $13.0 \%$ en las Humanidades, ampliamente despegadas del resto.

\section{Conclusión}

Esta investigación permite sostener que para la producción científica uruguaya las áreas de conocimiento presentan un comportamiento particular en relación a la publicación de los resultados de investigación. Estas particularidades deben considerarse muy especialmente a la hora de diseñar políticas de evaluación, fortalecimiento y acceso a los resultados de la investigación científica, que muchas veces privilegian la publicación en revistas de alto impacto descuidando otros canales de comunicación.

Se pueden identificar dos patrones claramente diferenciados que conforman dos grupos, por una parte el de las Ciencias Sociales y Humanidades y por otro el de las Ciencias Naturales y Exactas. El primer grupo se caracteriza por el predominio de la publicación en monografías (incluyendo los capítulos), en idioma español, de autoría individual, publicando en la región, y por una menor visibilidad internacional. Se destaca también la importante proporción de artículos publicados en revistas no arbitradas. Mientras que en el segundo grupo se privilegian los artículos en inglés y de autoría colectiva en revistas arbitradas, internacionales e indizadas. Las otras tres áreas tienen un comportamiento 
que las sitúa entre estos dos grupos, aunque en principio parecen más cercanas al segundo. No obstante, en algunos aspectos se diferencian de ambos, como por ejemplo la importancia de las ponencias publicadas, especialmente en el área de Ingeniería y Tecnología, y el peso del idioma español para las Ciencias Agrícolas y Médicas, entre otros.

Esta situación justifica el tipo de investigación y la fuente de datos utilizada, que permite estudiar la producción científica en su conjunto. Asimismo las características de la fuente utilizada limita la posibilidad de hacer comparaciones con trabajos anteriores, que generalmente parten de las bases de datos internacionales. Se concluye que los estudios como el presente resultan en una alternativa interesante y complementaria a los análisis bibliométricos realizados a partir de bases de datos comerciales. Estos últimos también son necesarios, y aún poco desarrollados en Uruguay, al permitir estudiar otros aspectos como impacto, visibilidad y colaboración.

Paralelamente y dada las limitaciones de los CV resultaría fundamental desarrollar herramientas de registro, conservación y acceso a la producción científica nacional que permitiesen su identificación, recuperación y estudio sistemático, y/o implementar mecanismos de mejora de la calidad de los datos de los CV.

Por otro lado, la investigación en curso muestra la necesidad de estudios que, atendiendo a las diferencias entre áreas, logren comprender la complejidad y variedad de canales y medios utilizados por los investigadores para publicar (en el sentido amplio de hacer público) los productos de su trabajo, y cómo se relaciona la sociedad con este conocimiento generado en ámbitos académicos.

\section{Referencias}

Aguirre-Ligüera, N. Aproximación al estudio de la producción científica uruguaya incluida en la Web of Science entre 2000 y 2009. Informatio, n.14/16, p.80-98, 2009-2011.

Bernal, J.D. Historia social de la ciencia l: la ciencia en la historia. Barcelona: Península, 1967.

Björk, B.C. A lifecicle model of a scientific communication process. Learned Publishing, v.18, n.3, p.165-176, 2005.

Bordons, M. et al. Perfil de actividad científica de las universidades españolas en cuatro áreas temáticas. Revista Española de Documentación Científica, v.33, n.1, p.9-33, 2010.

Borgman, C. Bibliometrics and scholarly communication: editor's introduction. Comunication Research, v.16, n.4, p.583-599, 1989.

Cañibano, C.; Bozeman, B. Curriculum vitae method in science policy and research evaluation: The state-of-the-art. Research Evaluation, v.18, n.2, p.86-94, 2009.

De Filippo, D. et al. El papel de las bases de datos institucionales en el análisis de la actividad científica de las universidades. Revista Española de Documentación Científica, v.34, n.2, p.165-189, 2011.

Dietz, J.S. et al. Using the curriculum vita to study the career paths of scientists and engineers: An exploratory assessment. Scientometrics, v.49, n.3, p.419-442, 2000

Fernández, M.; Frank, C.; Pitaluga, L. El conocimiento científico uruguayo en revistas internacionales 1981-2002. Montevideo: Instituto de Economía, 2005.

Gantman, E.R. La productividad científica argentina en ciencias sociales: economía, psicología, sociología y ciencia política en el CONICET (2004-2008). Revista Española de Documentación Cientifica, v.34, n.3, p.408-425, 2011.

Garvey, W.D.; Griffith, B.C. Communication and information processing within scientific disciplines: Empirical findings for Psychology. Information Storage and Retrieval, v.8, n.3, p.123-136, 1972.

Glanzel, W.; Schoepflin, U. A bibliometric study of reference literature in sciences and social science. Information Processing \& Management, v.35, n.1, p.31-44, 1999.

Gómez, N.D.; Arias, O.M. El cambio de paradigma en la comunicación científica. Información, Cultura y Sociedad, n.6, p.93-102, 2002. Disponible en: <http://www.scielo.org.ar>. Acceso en: 23 abr. 2012.

Hicks, D. The difficulty of achieving full coverage of international social science literature and the bibliometric consequences. Scientometrics, v.44, n.2, p.193-215, 1999.

Hurd, J.M. The transformation of scientific communication: A model for 2020. Journal of the American Society for Information Science, v.51, n.14, p.1279-1283, 2000.

López-Borrull, A. Física vs química: dos modelos de publicación científica. El profesional de la Información, v.21, n.2, p.167-172, 2012.

Maltrás Barba, B. Los indicadores bibliométricos: fundamentos y aplicación al análisis de la ciencia. Gijón, ES: Trea, 2003.

Miguel, S.; Gomez, N.-D.; Bongiovani, P. Acceso abierto real y potencial a la producción científica de un país: el caso argentino. El profesional de la Información, v.21, n.2, p.146153, 2012. Disponible en: <http://dx.doi.org/10.3145/ epi.2012.mar.04>. Acceso en: 23 abr. 2012. 
Rovira, L. ¿Hacia una evaluación métrica de la investigación en las humanidades y en las ciencias sociales? In: Ibarra, A.; Castro, J.; Barrenechea, J. (Ed.). La evaluación de la actividad científica en ciencias sociales y humanidades. Bilbao: Universidad del País Vasco, 2006. p.31-52.

Russell, J.M. La comunicación científica a comienzos del siglo XXI. Revista Internacional deficiencias Sociales, v.168, 2001. Disponible en: <http://oei.es/salactsi/rusell.pdf > . Acceso en: 22 abr. 2013.

Russell, J.M.; Liberman, S. Desarrollo de las bases de un modelo de comunicación de la producción científica de la Universidad Nacional Autónoma de México (UNAM). Revista Española de Documentación Científica, v.25, n.4, p.361-370, 2002

Sancho, R. Indicadores bibliométricos utilizados en la evaluación de la ciencia y la tecnología: revisión bibliográfica. Revista Española de Documentación Científica, v.13, n.3/4 p.842-865, 1990.
Sanz Casado, E.; Conforti, N. Análisis de la actividad científica de la Facultad de Humanidades de la Universidad de Mar del Plata, durante el período 1998-2001. Revista Española de Documentación Científica, v.28, n.2, p.196-205, 2005.

Targino, M.G. Produção intelectual, produção científica, produção acadêmica: facetas de uma mesma moeda? In: Gonçalves Curty, R. (Org.). Produção intelectual no ambiente acadêmico. Londrina: Universidade Estadual de Londrina, 2010, p.31-45. Disponível em: <http://www.uel.br/pos/mestrado informacao/pages/arquivos/Producao_Intelectual.pdf>. Acesso em: 14 fev. 2012.

Van Raan, A.F.J. Scientometrics: State of the art. Scientometrics, v.38, n.1, p.205-218, 1997.

Uruguay. Gabinete Ministerial de la Innovación. Informe a la sociedad: ciencia, tecnología e innovación en los últimos años. Montevideo: DICyT, 2012 

